\title{
INEQUALITIES FOR POWER SERIES WITH NONNEGATIVE COEFFICIENTS VIA REVERSES OF JENSEN INEQUALITY
}

\author{
SEver S. Dragomir
}

\begin{abstract}
Some inequalities for power series with nonnegative coefficients via a reverse of Jensen inequality obtained by Dragomir and Ionescu in 1994 are given. Applications for some fundamental functions defined by power series are also provided.
\end{abstract}

\section{Introduction}

In 1994, S. S. Dragomir and N. M. I on escu obtained the following reverse of Jensen's discrete inequality:

Let $\Phi: I \rightarrow \mathbb{R}$ be a differentiable convex function on the interior $\stackrel{\circ}{I}$ of the interval $I$. If $x_{i} \in \stackrel{\circ}{I}$ and $w_{i} \geq 0(i=1, \ldots, n)$ with $W_{n}:=\sum_{i=1}^{n} w_{i}=1$, then one has the inequality:

$$
0 \leq \sum_{i=1}^{n} w_{i} \Phi\left(x_{i}\right)-\Phi\left(\sum_{i=1}^{n} w_{i} x_{i}\right) \leq \sum_{i=1}^{n} w_{i} \Phi^{\prime}\left(x_{i}\right) x_{i}-\sum_{i=1}^{n} w_{i} \Phi^{\prime}\left(x_{i}\right) \sum_{i=1}^{n} w_{i} x_{i} .
$$

In order to improve Grüss' discrete inequality, P. C e r o n e and S. S. D r a g o m i r established in 2002 the following result [1]:

$$
\begin{aligned}
\left|\sum_{i=1}^{n} w_{i} a_{i} b_{i}-\sum_{i=1}^{n} w_{i} a_{i} \sum_{i=1}^{n} w_{i} b_{i}\right| & \leq \frac{1}{2}(A-a) \sum_{i=1}^{n} w_{i}\left|b_{i}-\sum_{j=1}^{n} w_{j} b_{j}\right| \\
& \leq \frac{1}{2}(A-a)\left[\sum_{i=1}^{n} w_{i} b_{i}^{2}-\left(\sum_{i=1}^{n} w_{i} b_{i}\right)^{2}\right]^{1 / 2}
\end{aligned}
$$

provided $-\infty<a \leq a_{i} \leq A<\infty$, and $w_{i} \geq 0(i=1, \ldots, n)$ with $W_{n}:=\sum_{i=1}^{n} w_{i}=1$.

(C) 2016 Mathematical Institute, Slovak Academy of Sciences.

2010 Mathematics Subject Classification: 26D15, 26 D10.

Keyw ords: power series, Jensen's inequality, reverse of Jensen's inequality. 
In addition, if $-\infty<b \leq b_{i} \leq B<\infty,(i=1, \ldots, n)$, then we have the string of inequalities

$$
\begin{aligned}
& \left|\sum_{i=1}^{n} w_{i} a_{i} b_{i}-\sum_{i=1}^{n} w_{i} a_{i} \sum_{i=1}^{n} w_{i} b_{i}\right| \\
& \leq \frac{1}{2}(A-a)\left[\sum_{i=1}^{n} w_{i} b_{i}^{2}-\left(\sum_{i=1}^{n} w_{i} b_{i}\right)^{2}\right]^{1 / 2} \leq \frac{1}{4}(A-a)(B-b) .
\end{aligned}
$$

Utilising these results, we observe that if $\Phi$ is differentiable convex on a finite interval, say $[m, M]$, then we have the inequalities:

$$
\begin{aligned}
0 & \leq \sum_{i=1}^{n} w_{i} \Phi\left(x_{i}\right)-\Phi\left(\sum_{i=1}^{n} w_{i} x_{i}\right) \\
& \leq \sum_{i=1}^{n} w_{i} \Phi^{\prime}\left(x_{i}\right) x_{i}-\sum_{i=1}^{n} w_{i} \Phi^{\prime}\left(x_{i}\right) \sum_{i=1}^{n} w_{i} x_{i} \\
& \leq \frac{1}{2}(M-m) \sum_{i=1}^{n} w_{i}\left|\Phi^{\prime}\left(x_{i}\right)-\sum_{j=1}^{n} w_{j} \Phi^{\prime}\left(x_{j}\right)\right| \\
& \leq \frac{1}{2}(M-m)\left[\sum_{i=1}^{n} w_{i}\left[\Phi^{\prime}\left(x_{i}\right)\right]^{2}-\left(\sum_{i=1}^{n} w_{i} \Phi^{\prime}\left(x_{i}\right)\right)^{2}\right]^{1 / 2}
\end{aligned}
$$

for $x_{i} \in(m, M)(i=1, \ldots, n)$.

If the lateral derivatives $\Phi_{+}^{\prime}(m)$ and $\Phi_{-}^{\prime}(M)$ are finite, then we also have

$$
\begin{aligned}
0 & \leq \sum_{i=1}^{n} w_{i} \Phi\left(x_{i}\right)-\Phi\left(\sum_{i=1}^{n} w_{i} x_{i}\right) \\
& \leq \frac{1}{2}\left[\Phi_{-}^{\prime}(M)-\Phi_{+}^{\prime}(m)\right] \sum_{i=1}^{n} w_{i}\left|x_{i}-\sum_{j=1}^{n} w_{j} x_{j}\right| \\
& \leq \frac{1}{2}\left[\Phi_{-}^{\prime}(M)-\Phi_{+}^{\prime}(m)\right]\left[\sum_{i=1}^{n} w_{i} x_{i}^{2}-\left(\sum_{i=1}^{n} w_{i} x_{i}\right)^{2}\right]^{1 / 2} \\
& \leq \frac{1}{4}(M-m)\left[\Phi_{-}^{\prime}(M)-\Phi_{+}^{\prime}(m)\right]
\end{aligned}
$$

for $x_{i} \in[m, M](i=1, \ldots, n)$.

In the recent paper [9], by the use of a refinement of Young's inequality, the authors proved the following result: 
Let $f(z)=\sum_{n=0}^{\infty} a_{n} z^{n}$ be a power series with nonnegative coefficients and convergent on the open disk $D(0, R)$ with $R>0$ or $R=\infty$. If $y, z, z^{\nu} y^{1-\nu}$, $z^{1-\nu} y^{\nu} \in(0, R)$ and $\nu \in[0,1]$, then we have the inequalities:

$$
\begin{aligned}
& 2 \min \{\nu, 1-\nu\}\left[f(y) f(z)-f^{2}(\sqrt{y z})\right] \\
& \leq f(y) f(z)-f\left(z^{\nu} y^{1-\nu}\right) f\left(z^{1-\nu} y^{\nu}\right) \\
& \leq 2 \max \{\nu, 1-\nu\}\left[f(y) f(z)-f^{2}(\sqrt{y z})\right]
\end{aligned}
$$

or, equivalently,

$$
\begin{aligned}
& 2 \min \{\nu, 1-\nu\}\left[f\left(u^{2}\right) f\left(t^{2}\right)-f^{2}(u t)\right] \\
& \leq f\left(u^{2}\right) f\left(t^{2}\right)-f\left(u^{2 \nu} t^{2(1-\nu)}\right) f\left(t^{2(1-\nu)} u^{2 \nu}\right) \\
& \leq 2 \max \{\nu, 1-\nu\}\left[f\left(u^{2}\right) f\left(t^{2}\right)-f^{2}(u t)\right],
\end{aligned}
$$

provided $u^{2}, t^{2}, u^{2 \nu} t^{2(1-\nu)}, t^{2(1-\nu)} u^{2 \nu} \in(0, R)$ and $\nu \in[0,1]$.

For other recent results for power series with nonnegative coefficients, see [9] and [10]. For more results on power series inequalities, see [2] and [5]- 8].

Motivated by the above results and utilizing a reverse of Jensen inequality obtained by S. S. Dragomir and N. M. I onescu in 1994, we provide in this paper other inequalities for power series with nonnegative coefficients. Applications for some fundamental functions are given as well.

\section{Power inequalities}

The most important power series with nonnegative coefficients are:

$$
\begin{array}{rlrl}
\exp (z) & =\sum_{n=0}^{\infty} \frac{1}{n !} z^{n}, & & z \in \mathbb{C}, \\
\frac{1}{1-z} & =\sum_{n=0}^{\infty} z^{n}, & & z \in D(0,1), \\
\ln \frac{1}{1-z} & =\sum_{n=1}^{\infty} \frac{1}{n} z^{n}, & & z \in D(0,1), \\
\cosh z & =\sum_{n=0}^{\infty} \frac{1}{(2 n) !} z^{2 n}, & & \\
\sinh z & =\sum_{n=0}^{\infty} \frac{1}{(2 n+1) !} z^{2 n+1}, & z \in \mathbb{C} .
\end{array}
$$


Other important examples of functions as power series representations with nonnegative coefficients are:

$$
\begin{array}{cc}
\frac{1}{2} \ln \left(\frac{1+z}{1-z}\right)=\tanh ^{-1}(z)=\sum_{n=1}^{\infty} \frac{1}{2 n-1} z^{2 n-1}, & z \in D(0,1), \\
\sin ^{-1}(z)=\sum_{n=0}^{\infty} \frac{\Gamma\left(n+\frac{1}{2}\right)}{\sqrt{\pi}(2 n+1) n !} z^{2 n+1}, & z \in D(0,1), \\
{ }_{2} F_{1}(\alpha, \beta, \gamma, z):=\sum_{n=0}^{\infty} \frac{\Gamma(n+\alpha) \Gamma(n+\beta) \Gamma(\gamma)}{n ! \Gamma(\alpha) \Gamma(\beta) \Gamma(n+\gamma)} z^{n}, \alpha, \beta, \gamma>0, & z \in D(0,1),
\end{array}
$$

where $\Gamma$ is Gamma function.

The following result for powers holds:

Theorem 1. Let $f(z)=\sum_{n=0}^{\infty} a_{n} z^{n}$ be a power series with nonnegative coefficients and convergent on the open disk $D(0, R)$ with $R>0$ or $R=\infty$. If $p \geq 1$, $0<\alpha<R$ and $x>0$ with $\alpha x^{p}, \alpha x^{p-1}<R$, then

$$
0 \leq \frac{f\left(\alpha x^{p}\right)}{f(\alpha)}-\left[\frac{f(\alpha x)}{f(\alpha)}\right]^{p} \leq p\left[\frac{f\left(\alpha x^{p}\right)}{f(\alpha)}-\frac{f\left(\alpha x^{p-1}\right)}{f(\alpha)} \frac{f(\alpha x)}{f(\alpha)}\right]
$$

Moreover, if $0<x \leq 1$, then

$$
\begin{aligned}
0 & \leq \frac{f\left(\alpha x^{p}\right)}{f(\alpha)}-\left[\frac{f(\alpha x)}{f(\alpha)}\right]^{p} \\
& \leq p\left[\frac{f\left(\alpha x^{p}\right)}{f(\alpha)}-\frac{f\left(\alpha x^{p-1}\right)}{f(\alpha)} \frac{f(\alpha x)}{f(\alpha)}\right] \\
& \leq \frac{1}{2} p\left(\frac{f\left(\alpha x^{2(p-1)}\right)}{f(\alpha)}-\left[\frac{f\left(\alpha x^{p-1}\right)}{f(\alpha)}\right]^{2}\right)^{1 / 2} \leq \frac{1}{4} p
\end{aligned}
$$

and

$$
\begin{aligned}
0 & \leq \frac{f\left(\alpha x^{p}\right)}{f(\alpha)}-\left[\frac{f(\alpha x)}{f(\alpha)}\right]^{p} \\
& \leq p\left[\frac{f\left(\alpha x^{p}\right)}{f(\alpha)}-\frac{f\left(\alpha x^{p-1}\right)}{f(\alpha)} \frac{f(\alpha x)}{f(\alpha)}\right] \\
& \leq \frac{1}{2} p\left(\frac{f\left(\alpha x^{2}\right)}{f(\alpha)}-\left[\frac{f(\alpha x)}{f(\alpha)}\right]^{2}\right)^{1 / 2} \leq \frac{1}{4} p .
\end{aligned}
$$


Proof. If we write the inequality (1.1) for the convex function $\Phi:[0, \infty) \rightarrow$ $[0, \infty), \Phi(x)=x^{p}, p \geq 1$, then we have

$$
\begin{aligned}
0 & \leq \sum_{i=1}^{n} w_{i} x_{i}^{p}-\left(\sum_{i=1}^{n} w_{i} x_{i}\right)^{p} \\
& \leq p\left(\sum_{i=1}^{n} w_{i} x_{i}^{p}-\sum_{i=1}^{n} w_{i} x_{i}^{p-1} \sum_{i=1}^{n} w_{i} x_{i}\right)
\end{aligned}
$$

for any $w_{i}, x_{i} \geq 0(i=1, \ldots, n)$ with $\sum_{i=1}^{n} w_{i}=1$.

If $0<\alpha<R$ and $k \geq 1$, then by (2.6) we have

$$
\begin{aligned}
0 \leq & \frac{1}{\sum_{j=0}^{k} a_{j} \alpha^{j}} \sum_{j=0}^{k} a_{j} \alpha^{j}\left(x^{p}\right)^{j}-\left(\frac{1}{\sum_{j=0}^{k} a_{j} \alpha^{j}} \sum_{j=0}^{k} a_{j} \alpha^{j} x^{j}\right)^{p} \\
\leq p & {\left[\frac{1}{\sum_{j=0}^{k} a_{j} \alpha^{j}} \sum_{j=0}^{k} a_{j} \alpha^{j}\left(x^{p}\right)^{j}\right.} \\
& \left.-\frac{1}{\sum_{j=0}^{k} a_{j} \alpha^{j}} \sum_{j=0}^{k} a_{j} \alpha^{j}\left(x^{p-1}\right)^{j} \frac{1}{\sum_{j=0}^{k} a_{j} \alpha^{j}} \sum_{j=0}^{k} a_{j} \alpha^{j} x^{j}\right] .
\end{aligned}
$$

Since all series whose partial sums involved in the inequality (2.7) are convergent, then by letting $k \rightarrow \infty$ in (2.7) we deduce (2.3).

Now, if $x_{i} \in[m, M] \subset[0, \infty),(i=1, \ldots, n)$, then by (1.4) for the convex function $\Phi:[0, \infty) \rightarrow[0, \infty), \Phi(x)=x^{p}, p \geq 1$ we have

$$
\begin{aligned}
0 & \leq \sum_{i=1}^{n} w_{i} x_{i}^{p}-\left(\sum_{i=1}^{n} w_{i} x_{i}\right)^{p} \\
& \leq p\left(\sum_{i=1}^{n} w_{i} x_{i}^{p}-\sum_{i=1}^{n} w_{i} x_{i}^{p-1} \sum_{i=1}^{n} w_{i} x_{i}\right) \\
& \leq \frac{1}{2} p(M-m) \sum_{i=1}^{n} w_{i}\left|x_{i}^{p-1}-\sum_{j=1}^{n} w_{j} x_{j}^{p-1}\right| \\
& \leq \frac{1}{2} p(M-m)\left[\sum_{i=1}^{n} w_{i} x_{i}^{2(p-1)}-\left(\sum_{i=1}^{n} w_{i} x_{i}^{p-1}\right)^{2}\right]^{1 / 2} \\
& \leq \frac{1}{4} p(M-m)\left(M^{p-1}-m^{p-1}\right) .
\end{aligned}
$$


If $0<x \leq 1$, then $0<x^{j} \leq 1$ for $j=0, \ldots, k$, and by (2.8), we have

$$
\begin{aligned}
0 & \leq \frac{1}{\sum_{j=0}^{k} a_{j} \alpha^{j}} \sum_{j=0}^{k} a_{j} \alpha^{j}\left(x^{p}\right)^{j}-\left(\frac{1}{\sum_{j=0}^{k} a_{j} \alpha^{j}} \sum_{j=0}^{k} a_{j} \alpha^{j} x^{j}\right)^{p} \\
\leq & p\left[\frac{1}{\sum_{j=0}^{k} a_{j} \alpha^{j}} \sum_{j=0}^{k} a_{j} \alpha^{j}\left(x^{p}\right)^{j}\right. \\
& \left.-\frac{1}{\sum_{j=0}^{k} a_{j} \alpha^{j}} \sum_{j=0}^{k} a_{j} \alpha^{j}\left(x^{p-1}\right)^{j} \frac{1}{\sum_{j=0}^{k} a_{j} \alpha^{j}} \sum_{j=0}^{k} a_{j} \alpha^{j} x^{j}\right] \\
\leq & \frac{1}{2} p \\
& \times\left[\frac{1}{\sum_{j=0}^{k} a_{j} \alpha^{j}} \sum_{j=0}^{k} a_{j} \alpha^{j}\left[x^{2(p-1)}\right]^{j}-\left(\frac{1}{\sum_{j=0}^{k} a_{j} \alpha^{j}} \sum_{j=0}^{k} a_{j} \alpha^{j}\left(x^{p-1}\right)^{j}\right)^{2}\right]^{1 / 2} \\
\leq & \frac{1}{4} p .
\end{aligned}
$$

Since all series whose partial sums involved in the inequality (2.9) are convergent, then by letting $k \rightarrow \infty$ in (2.9) we deduce (2.4).

Now, if $x_{i} \in[m, M] \subset[0, \infty),(i=1, \ldots, n)$, then by (1.5) for the convex function $\Phi:[0, \infty) \rightarrow[0, \infty), \Phi(x)=x^{p}, p \geq 1$ we have

$$
\begin{aligned}
0 & \leq \sum_{i=1}^{n} w_{i} x_{i}^{p}-\left(\sum_{i=1}^{n} w_{i} x_{i}\right)^{p} \\
& \leq p\left(\sum_{i=1}^{n} w_{i} x_{i}^{p}-\sum_{i=1}^{n} w_{i} x_{i}^{p-1} \sum_{i=1}^{n} w_{i} x_{i}\right) \\
& \leq \frac{1}{2} p\left(M^{p-1}-m^{p-1}\right) \sum_{i=1}^{n} w_{i}\left|x_{i}-\sum_{j=1}^{n} w_{j} x_{j}\right| \\
& \leq \frac{1}{2} p\left(M^{p-1}-m^{p-1}\right)\left[\sum_{i=1}^{n} w_{i} x_{i}^{2}-\left(\sum_{i=1}^{n} w_{i} x_{i}\right)^{2}\right]^{1 / 2} \\
& \leq \frac{1}{4} p(M-m)\left(M^{p-1}-m^{p-1}\right) .
\end{aligned}
$$

Finally, by utilizing a similar argument as above, we obtain the inequality (2.5). The details are omitted. 
Remark 1. We observe that the second inequality in (2.3) is equivalent to

$$
\frac{f(\alpha x)}{f(\alpha)}\left(p \frac{f\left(\alpha x^{p-1}\right)}{f(\alpha)}-\left[\frac{f(\alpha x)}{f(\alpha)}\right]^{p-1}\right) \leq(p-1) \frac{f\left(\alpha x^{p}\right)}{f(\alpha)}
$$

or to

$$
f(\alpha x)\left(p f\left(\alpha x^{p-1}\right)[f(\alpha)]^{p-2}-[f(\alpha x)]^{p-1}\right) \leq(p-1) f\left(\alpha x^{p}\right)[f(\alpha)]^{p-1},
$$

provided that $p \geq 1,0<\alpha<R$ and $x>0$ with $\alpha x^{p}, \alpha x^{p-1}<R$.

Moreover, if $0<x \leq 1$, then from (2.4) we have

$$
\left[\frac{f(\alpha x)}{f(\alpha)}\right]^{p} \leq \frac{f\left(\alpha x^{p}\right)}{f(\alpha)} \leq \frac{1}{4} p+\left[\frac{f(\alpha x)}{f(\alpha)}\right]^{p} .
$$

Taking the power $1 / p$ and using the inequality $(a+b)^{1 / p} \leq a^{1 / p}+b^{1 / p}, p \geq 1$, we get

$$
0 \leq\left[f\left(\alpha x^{p}\right)\right]^{1 / p}[f(\alpha)]^{1-\frac{1}{p}}-f(\alpha x) \leq \frac{1}{4^{1 / p}} p^{1 / p} f(\alpha) .
$$

Corollary 1. Let $f(z)=\sum_{n=0}^{\infty} a_{n} z^{n}$ be a power series with nonnegative coefficients and convergent on the open disk $D(0, R)$ with $R>0$ or $R=\infty$. If $p>1$, $\frac{1}{p}+\frac{1}{q}=1$ and $u, v>0$ with $v^{p} \leq u^{q}<R$, then

$$
\left[\frac{f(u v)}{f\left(u^{q}\right)}\right]^{p} \leq \frac{f\left(v^{p}\right)}{f\left(u^{q}\right)} \leq \frac{1}{4} p+\left[\frac{f(u v)}{f\left(u^{q}\right)}\right]^{p}
$$

and

$$
0 \leq\left[f\left(v^{p}\right)\right]^{1 / p}\left[f\left(u^{q}\right)\right]^{1 / q}-f(u v) \leq \frac{1}{4^{1 / p}} p^{1 / p} f\left(u^{q}\right) .
$$

P r o o f. Follows by taking into (2.13) and (2.14) $\alpha=u^{q}$ and $x=\frac{v}{u^{q / p}}$. The details are omitted.

EXAMPLE 1.

a) If we write the inequalities (2.4) and (2.5) for the function $\frac{1}{1-z}=\sum_{n=0}^{\infty} z^{n}$, $z \in D(0,1)$, then we have

$$
\begin{aligned}
0 & \leq \frac{1-\alpha}{1-\alpha x^{p}}-\left(\frac{1-\alpha}{1-\alpha x}\right)^{p} \\
& \leq p\left[\frac{1-\alpha}{1-\alpha x^{p}}-\frac{(1-\alpha)^{2}}{\left(1-\alpha x^{p-1}\right)(1-\alpha x)}\right] \\
& \leq \frac{1}{2} p\left[\frac{1-\alpha}{1-\alpha x^{2(p-1)}}-\left(\frac{1-\alpha}{1-\alpha x^{p-1}}\right)^{2}\right]^{1 / 2} \leq \frac{1}{4} p
\end{aligned}
$$


and

$$
\begin{aligned}
0 & \leq \frac{1-\alpha}{1-\alpha x^{p}}-\left(\frac{1-\alpha}{1-\alpha x}\right)^{p} \\
& \leq p\left[\frac{1-\alpha}{1-\alpha x^{p}}-\frac{(1-\alpha)^{2}}{\left(1-\alpha x^{p-1}\right)(1-\alpha x)}\right] \\
& \leq \frac{1}{2} p\left[\frac{1-\alpha}{1-\alpha x^{2}}-\left(\frac{1-\alpha}{1-\alpha x}\right)^{2}\right]^{1 / 2} \leq \frac{1}{4} p
\end{aligned}
$$

for any $\alpha, x \in(0,1)$ and $p \geq 1$.

b) If we write the inequalities (2.4) and (2.5) for the function $\exp z=\sum_{n=0}^{\infty} \frac{1}{n !} z^{n}, z \in \mathbb{C}$, then we have

$$
\begin{aligned}
0 & \leq \exp \left[\alpha\left(x^{p}-1\right)\right]-\exp [p \alpha(x-1)] \\
& \leq p\left[\exp \left[\alpha\left(x^{p}-1\right)\right]-\exp \left[\alpha\left(x^{p-1}+x-2\right)\right]\right] \\
& \leq \frac{1}{2} p\left(\exp \left[\alpha\left(x^{2(p-1)}-1\right)\right]-\exp \left[2 \alpha\left(x^{p-1}-1\right)\right]\right)^{1 / 2} \leq \frac{1}{4} p
\end{aligned}
$$

and

$$
\begin{aligned}
0 & \leq \exp \left[\alpha\left(x^{p}-1\right)\right]-\exp [p \alpha(x-1)] \\
& \leq p\left[\exp \left[\alpha\left(x^{p}-1\right)\right]-\exp \left[\alpha\left(x^{p-1}+x-2\right)\right]\right] \\
& \leq \frac{1}{2} p\left(\exp \left[\alpha\left(x^{2}-1\right)\right]-\exp [2 \alpha(x-1)]\right)^{1 / 2} \leq \frac{1}{4} p .
\end{aligned}
$$

for any $\alpha, p>0$ and $x \in(0,1)$.

\section{Exponential inequalities}

The following exponential inequality holds:

TheOREM 2. Let $f(z)=\sum_{n=0}^{\infty} a_{n} z^{n}$ be a power series with nonnegative coefficients and convergent on the open disk $D(0, R)$ with $R>0$ or $R=\infty$. If $0<\alpha<R$ and $x, \beta \in \mathbb{R}$ with $\alpha \exp (\beta x)<R$, then

$$
\begin{aligned}
0 & \leq \frac{f(\alpha \exp (\beta x))}{f(\alpha)}-\exp \left[\frac{\alpha \beta x f^{\prime}(\alpha)}{f(\alpha)}\right] \\
& \leq \alpha \beta x\left[\frac{\exp (\beta x) f^{\prime}(\alpha \exp (\beta x))}{f(\alpha)}-\frac{f(\alpha \exp (\beta x))}{f(\alpha)} \frac{f^{\prime}(\alpha)}{f(\alpha)}\right] .
\end{aligned}
$$


Moreover, if $x \leq 0, \beta>0$ with $\exp (\beta x)<R$ and $0<\alpha<R$, then

$$
\begin{aligned}
0 & \leq \frac{f(\alpha \exp (\beta x))}{f(\alpha)}-\exp \left[\frac{\alpha \beta x f^{\prime}(\alpha)}{f(\alpha)}\right] \\
& \leq \alpha \beta x\left[\frac{\exp (\beta x) f^{\prime}(\alpha \exp (\beta x))}{f(\alpha)}-\frac{f(\alpha \exp (\beta x))}{f(\alpha)} \frac{f^{\prime}(\alpha)}{f(\alpha)}\right] \\
& \leq \frac{1}{2} \beta|x|\left[\frac{\alpha\left[f^{\prime}(\alpha)+\alpha f^{\prime \prime}(\alpha)\right]}{f(\alpha)}-\left(\frac{\alpha f^{\prime}(\alpha)}{f(\alpha)}\right)^{2}\right]^{1 / 2} .
\end{aligned}
$$

Proof. It is similar to the proof in Theorem 1 for the convex function $\Phi: \mathbb{R} \rightarrow$ $[0, \infty), \Phi(x)=\exp (\beta x)$ and the choice $x_{j}=j x, j \in\{0, \ldots, k\}$. The details are omitted.

EXAMPLE 2.

a) If we write the inequality (3.2) for the function $\frac{1}{1-z}=\sum_{n=0}^{\infty} z^{n}, z \in D(0,1)$, then we have for $x \leq 0, \beta>0$ and $0<\alpha<1$, that

$$
\begin{aligned}
0 & \leq \frac{1-\alpha}{1-\alpha \exp (\beta x)}-\exp \left(\frac{\alpha \beta x}{1-\alpha}\right) \\
& \leq \alpha \beta x\left[\frac{(1-\alpha) \exp (\beta x)}{(1-\alpha \exp (\beta x))^{2}}-\frac{1}{1-\alpha \exp (\beta x)}\right] \leq \frac{1}{2} \frac{\beta|x| \alpha^{1 / 2}}{1-\alpha} .
\end{aligned}
$$

b) If we write the inequality (3.1) for the function $\exp z=\sum_{n=0}^{\infty} \frac{1}{n !} z^{n}, z \in \mathbb{C}$, then we have

$$
\begin{aligned}
0 & \leq \exp (\alpha[\exp (\beta x)-1])-\exp (\alpha \beta x) \\
& \leq \alpha \beta x[\exp (\alpha[\exp (\beta x)-1]+\beta x)-\exp (\alpha[\exp (\beta x)-1])]
\end{aligned}
$$

for any $\alpha>0$ and $x \leq 0, \beta>0$.

\section{Logarithmic inequalities}

The following logarithmic inequality holds:

Theorem 3. Let $f(z)=\sum_{n=0}^{\infty} a_{n} z^{n}$ be a power series with nonnegative coefficients and convergent on the open disk $D(0, R)$ with $R>0$ or $R=\infty$. If $0<\alpha<R$, $p>0$ and $x>0$ with $\alpha x^{p}, \alpha x^{-p}<R$, then

$$
0 \leq \ln \left(\frac{f\left(\alpha x^{p}\right)}{f(\alpha)}\right)-p \frac{\alpha f^{\prime}(\alpha)}{f(\alpha)} \ln x \leq \frac{f\left(\alpha x^{p}\right)}{f(\alpha)} \frac{f\left(\alpha x^{-p}\right)}{f(\alpha)}-1
$$


Moreover, if $0<x \leq 1$ with $\alpha x^{p}, \alpha x^{-p}<R$, then

$$
\begin{aligned}
0 & \leq \ln \left(\frac{f\left(\alpha x^{p}\right)}{f(\alpha)}\right)-p \frac{\alpha f^{\prime}(\alpha)}{f(\alpha)} \ln x \leq \frac{f\left(\alpha x^{p}\right)}{f(\alpha)} \frac{f\left(\alpha x^{-p}\right)}{f(\alpha)}-1 \\
& \leq \frac{1}{2}\left[\frac{f\left(\alpha x^{-2 p}\right)}{f(\alpha)}-\left(\frac{f\left(\alpha x^{-p}\right)}{f(\alpha)}\right)^{2}\right]^{1 / 2} .
\end{aligned}
$$

Proof. It is similar to the proof in Theorem 1 for the convex function $\Phi:(0, \infty) \rightarrow \mathbb{R}, \Phi(x)=-\ln x$ and the choice $x_{j}=\left(x^{p}\right)^{j}, j \in\{0, \ldots, k\}$. The details are omitted.

Corollary 2. Let $f(z)=\sum_{n=0}^{\infty} a_{n} z^{n}$ be a power series with nonnegative coefficients and convergent on the open disk $D(0, R)$ with $R>0$ or $R=\infty$. If $p>1$, $\frac{1}{p}+\frac{1}{q}=1$ and $u, v>0$ with $v^{p}, u^{q}, \frac{u^{2 q}}{v^{p}}<R$, then

$$
0 \leq \ln \left(\frac{f\left(v^{p}\right)}{f\left(u^{q}\right)}\right)-\frac{u^{q} f^{\prime}\left(u^{q}\right)}{f\left(u^{q}\right)} \ln \frac{v^{p}}{u^{q}} \leq \frac{f\left(v^{p}\right)}{f\left(u^{q}\right)} \frac{f\left(\frac{u^{2 q}}{v^{p}}\right)}{f\left(u^{q}\right)}-1 .
$$

If $v^{p} \leq u^{q}<R$ and $\frac{u^{2 q}}{v^{p}}, \frac{u^{3 q}}{v^{2 p}}$, then

$$
\begin{aligned}
0 & \leq \ln \left(\frac{f\left(v^{p}\right)}{f\left(u^{q}\right)}\right)-\frac{u^{q} f^{\prime}\left(u^{q}\right)}{f\left(u^{q}\right)} \ln \frac{v^{p}}{u^{q}} \leq \frac{f\left(v^{p}\right)}{f\left(u^{q}\right)} \frac{f\left(\frac{u^{2 q}}{v^{p}}\right)}{f\left(u^{q}\right)}-1 \\
& \leq \frac{1}{2}\left[\frac{f\left(\frac{u^{3 q}}{v^{2 p}}\right)}{f\left(u^{q}\right)}-\left(\frac{f\left(\frac{u^{2 q}}{v^{p}}\right)}{f\left(u^{q}\right)}\right)^{2}\right]^{1 / 2} .
\end{aligned}
$$

P r o o f. Follows by taking $\alpha=u^{q}$ and $x=\frac{v}{u^{q / p}}$ into (4.1) and (4.2). The details are omitted.

EXAMPLE 3.

a) If we write the inequality (4.2) for the function $\frac{1}{1-z}=\sum_{n=0}^{\infty} z^{n}, z \in D(0,1)$, then we have for $0<\alpha, x<1$ and $p>0$ that

$$
\begin{aligned}
0 & \leq \ln \left(\frac{1-\alpha}{1-\alpha x^{p}}\right)-\frac{p \alpha}{1-\alpha} \ln x \\
& \leq \frac{(1-\alpha)^{2}}{\left(1-\alpha x^{p}\right)\left(1-\alpha x^{-p}\right)}-1 \\
& \leq \frac{1}{2}\left[\frac{1-\alpha}{1-\alpha x^{-2 p}}-\left(\frac{1-\alpha}{1-\alpha x^{-p}}\right)^{2}\right]^{1 / 2} .
\end{aligned}
$$


b) If we write the inequality (4.1) for the function $\exp z=\sum_{n=0}^{\infty} \frac{1}{n !} z^{n}, z \in \mathbb{C}$, then we have

$$
0 \leq \alpha\left(x^{p}-1\right)-p \alpha \ln x \leq \exp \left[\alpha\left(x^{p}+x^{-p}-2\right)\right]-1
$$

for $\alpha, p, x>0$.

The following logarithmic inequality also holds:

Theorem 4. Let $f(z)=\sum_{n=0}^{\infty} a_{n} z^{n}$ be a power series with nonnegative coefficients and convergent on the open disk $D(0, R)$ with $R>0$ or $R=\infty$. If $0<\alpha<R, p>0$ and $x>0$ with $\alpha x^{p}<R$, then

$$
\begin{aligned}
0 & \leq \frac{p \alpha x^{p} f^{\prime}\left(\alpha x^{p}\right)}{f(\alpha)} \ln x-\frac{f\left(\alpha x^{p}\right)}{f(\alpha)} \ln \left(\frac{f\left(\alpha x^{p}\right)}{f(\alpha)}\right) \\
& \leq p \alpha\left[\frac{x^{p} f^{\prime}\left(\alpha x^{p}\right)}{f(\alpha)}-\frac{f\left(\alpha x^{p}\right)}{f(\alpha)} \frac{f^{\prime}(\alpha)}{f(\alpha)}\right] \ln x
\end{aligned}
$$

or, equivalently

$$
\frac{p \alpha f^{\prime}(\alpha)}{f(\alpha)} \ln x \leq \ln \left(\frac{f\left(\alpha x^{p}\right)}{f(\alpha)}\right)
$$

i.e., the first inequality in (4.1).

Moreover, if $0<x \leq 1$ we also have

$$
\begin{aligned}
0 & \leq \frac{p \alpha x^{p} f^{\prime}\left(\alpha x^{p}\right)}{f(\alpha)} \ln x-\frac{f\left(\alpha x^{p}\right)}{f(\alpha)} \ln \left(\frac{f\left(\alpha x^{p}\right)}{f(\alpha)}\right) \\
& \leq p \alpha\left[\frac{x^{p} f^{\prime}\left(\alpha x^{p}\right)}{f(\alpha)}-\frac{f\left(\alpha x^{p}\right)}{f(\alpha)} \frac{f^{\prime}(\alpha)}{f(\alpha)}\right] \ln x \\
& \leq \frac{1}{2} p|\ln x|\left[\frac{\alpha\left[f^{\prime}(\alpha)+\alpha f^{\prime \prime}(\alpha)\right]}{f(\alpha)}-\left(\frac{\alpha f^{\prime}(\alpha)}{f(\alpha)}\right)^{2}\right]^{1 / 2}
\end{aligned}
$$

Proof. It is similar to the proof in Theorem 1 for the convex function $\Phi:(0, \infty) \rightarrow \mathbb{R}, \Phi(x)=x \ln x$, and the choice $x_{j}=\left(x^{p}\right)^{j}, j \in\{0, \ldots, k\}$. The details are omitted.

CoRollaRy 3. Let $f(z)=\sum_{n=0}^{\infty} a_{n} z^{n}$ be a power series with nonnegative coefficients and convergent on the open disk $D(0, R)$ with $R>0$ or $R=\infty$. 
If $p>1, \frac{1}{p}+\frac{1}{q}=1$ and $u, v>0$ with $v^{p} \leq u^{q}<R$, then

$$
\begin{aligned}
0 & \leq \frac{f\left(v^{p}\right)}{f\left(u^{q}\right)} \ln \left(\frac{f\left(u^{q}\right)}{f\left(v^{p}\right)}\right)-\frac{v^{p} f^{\prime}\left(v^{p}\right)}{f\left(u^{q}\right)} \ln \left(\frac{u^{q}}{v^{p}}\right) \\
& \leq u^{q}\left[\frac{f\left(v^{p}\right)}{f\left(u^{q}\right)} \frac{f^{\prime}\left(u^{q}\right)}{f\left(u^{q}\right)}-\frac{\frac{v^{p}}{u^{q}} f^{\prime}\left(v^{p}\right)}{f\left(u^{q}\right)}\right] \ln \left(\frac{u^{q}}{v^{p}}\right) \\
& \leq \frac{1}{2}\left[\frac{u^{q}\left[f^{\prime}\left(u^{q}\right)+u^{q} f^{\prime \prime}\left(u^{q}\right)\right]}{f\left(u^{q}\right)}-\left(\frac{u^{q} f^{\prime}\left(u^{q}\right)}{f\left(u^{q}\right)}\right)^{2}\right]^{1 / 2} \ln \left(\frac{u^{q}}{v^{p}}\right) .
\end{aligned}
$$

EXAMPLE 4. a) If we write the inequality (4.8) for the function $\frac{1}{1-z}=\sum_{n=0}^{\infty} z^{n}$, $z \in D(0,1)$, then we have for $\alpha, x \in(0,1)$ and $p>0$ that

$$
\begin{aligned}
0 & \leq \frac{p \alpha x^{p}(1-\alpha)}{\left(1-\alpha x^{p}\right)^{2}} \ln x-\frac{1-\alpha}{\left(1-\alpha x^{p}\right)} \ln \left(\frac{1-\alpha}{1-\alpha x^{p}}\right) \\
& \leq p \alpha\left[\frac{x^{p}(1-\alpha)}{\left(1-\alpha x^{p}\right)^{2}}-\frac{1}{1-\alpha x^{p}}\right] \ln x \\
& \leq \frac{1}{2} \frac{p \alpha^{1 / 2}}{1-\alpha}|\ln x| .
\end{aligned}
$$

b) If we write the inequality (4.8) for the function $\exp z=\sum_{n=0}^{\infty} \frac{1}{n !} z^{n}, z \in \mathbb{C}$, then we have

$$
\begin{aligned}
0 & \leq\left[p \alpha x^{p} \ln x-\alpha\left(x^{p}-1\right)\right] \exp \left[\alpha\left(x^{p}-1\right)\right] \\
& \leq p \alpha\left(x^{p}-1\right) \exp \left[\alpha\left(x^{p}-1\right)\right] \ln x \\
& \leq \frac{1}{2} p|\ln x| \alpha^{1 / 2}
\end{aligned}
$$

for $x \in(0,1)$ and $\alpha, p>0$.

Acknowledgements. The author would like to thank the anonymous referee for valuable recommendations that have been implemented in the final version of this paper.

\section{REFERENCES}

[1] CERONE, P.-DRAGOMIR, S. S.: A refinement of the Grüss inequality and applications, Tamkang J. Math. 38 (2007), 37-49. Preprint RGMIA Res. Rep. Coll. 5 (2002), Art. 14. http://rgmia.org/papers/v5n2/RGIApp.pdf

[2] CERONE, P.-DRAGOMIR, S. S.: Some applications of de Bruijn's inequality for power series, Integral Transforms Spec. Funct. 18 (2007), 387-396. 


\section{INEQUALITIES FOR POWER SERIES WITH NONNEGATIVE COEFFICIENTS}

[3] DRAGOMIR, S. S.: Discrete Inequalities of the Cauchy-Bunyakovsky-Schwarz Type, Nova Sci. Publ. Inc., Hauppauge, N.Y., 2004.

[4] DRAGOMIR, S. S.-IONESCU, N. M.: Some converse of Jensen's inequality and applications, Rev. Anal. Numér. Théor. Approx. 23 (1994), 71-78.

[5] IBRAHIM, A.-DRAGOMIR, S. S.: Power series inequalities via Buzano's result and applications, Integral Transforms Spec. Funct. 22 (2011), 867-878.

[6] IBRAHIM, A.-DRAGOMIR, S. S.: Power series inequalities via a refinement of Schwarz inequality, Integral Transforms Spec. Funct. 23 (2012), 769-78.

[7] IBRAHIM, A.-DRAGOMIR, S. S.: A survey on Cauchy-Bunyakovsky-Schwarz inequality for power series, in: Analytic Number Theory, Approximation Theory, and Special Functions (G. V. Milovanović and M. Th. Rassias, eds.), Springer, New York, 2013, pp. 247-295.

[8] IBRAHIM, A.-DRAGOMIR, S. S.-DARUS, M.: Some inequalities for power series with applications, Integral Transforms Spec. Funct. 24 (2013), 364-376.

[9] IBRAHIM, A.-DRAGOMIR, S. S.-DARUS, M.: Power series inequalities related to Young's inequality and applications, Integral Transforms Spec. Funct. 24 (2013), $700-714$.

[10] IBRAHIM, A.-DRAGOMIR, S. S.-DARUS, M.: Power series inequalities via Young's inequality with applications, J. Inequal. Appl. 2013, Article ID 314, 13 pp.

Mathematics

School of Engineering and Science

Victoria University

PO Box 14428

Melbourne City, MC 8001

AUSTRALIA

E-mail: sever.dragomir@vu.edu.au

School of Computational and

Applied Mathematics

University of the Witwatersrand

Private Bag 3

Johannesburg 2050

SOUTH AFRICA 